\title{
Developmental Status of the Potential Vaccines for the Mitigation of the COVID-19 Pandemic and a Focus on the Effectiveness of the Pfizer-BioNTech and Moderna mRNA Vaccines
}

\author{
Rashed Noor $^{1}$ (1) \\ Accepted: 18 February 2021 / Published online: 3 March 2021 \\ (C) The Author(s), under exclusive licence to Springer Nature Switzerland AG part of Springer Nature 2021
}

\begin{abstract}
Purpose of Review Along with the continued in silico-based studies for drug designing and repurposing followed by the corresponding cell culture studies, the ongoing clinical trials with some completed regarding finding the drug efficacy and the vaccine development against the severe acute respiratory coronavirus 2 (SARS-CoV-2) have been the most functional and indispensable issue during the current COVID-19 pandemic within 2020 and onward. The present review attempted to figure out the update on this effective vaccine and discussed the other promising vaccines.

Recent findings A range of investigations on the SARS-CoV-2 genomics, on its similarities with SARS-CoV-1, and with the Middle East respiratory syndrome coronavirus (MERS-CoV) have been accomplished and the host immune dodging mechanisms by the SARS-CoV-2 have been unraveled which in turn led the scientists around the world to work rigorously on the vaccine development. Working with various vaccine platforms so far revealed the efficacy of the mRNA-1273 vaccine as the most effective one as resulted through the clinical trials which resulted in $95 \%$ positive output.

Summary Although currently commercialized mRNA-1273 vaccine appears to be effective, still several points are to be pondered regarding the sustainability of vaccine efficacy against the rising variants of SARS-CoV-2.
\end{abstract}

Keywords COVID-19 pandemic $\cdot$ SARS-CoV-2 $\cdot$ mRNA vaccines

\section{Introduction}

Respiratory viral infections and the associated pandemic have quite a long history of more than 130 years. The first dreadful pandemic, the Russian influenza (1889-1892) caused by the influenza A virus subtype H2N2, accounted for more than a million of deaths worldwide, and within the 30 years ahead, in 1918-1920, the Spanish flu pandemic took more than 50 million lives [1]. After 100 years of Spanish flu, the ongoing COVID-19 pandemic (originating from bats in Wuhan, China, at the end of December 2019 lasting till date) caused by the severe acute respiratory coronavirus 2 (SARS-CoV-2)

Rashed Noor

rashednoor@iub.edu.bd

1 Department of Life Sciences (DLS), School of Environment and Life Sciences (SELS), Independent University, Bangladesh (IUB), Plot 16, Block B, Aftabuddin Ahmed Road, Bashundhara, Dhaka 1229, Bangladesh has appeared to be the horrific viral infection threat to the global public health, already causing 1,744,235 deaths out of $78,604,532$ confirmed cases worldwide with a prodigious increase in the number of infected individuals within a year [1, 2]. Indeed, till the prevalence of coronaviruses (SARS-CoV in 2002-2003, causing 774 deaths in 24 countries, and the Middle East respiratory syndrome coronavirus or MERS$\mathrm{CoV}$ endemic in 2012, followed by the current SARS-CoV2 instigated COVID-19 pandemic), in 1957-1959, the Asian influenza caused around 0.7 million deaths, and the causative virus subtype H2N2 lasted for 11 years ending up to Hong Kong influenza in 1968 causing 1 million deaths [1].

Regarding the first step for the coronavirus pathogenicity, this is already very well known that SARS-CoV-2 spike (S) protein $\mathrm{S} 1$ subunit in trimer (which associates at the top of $\mathrm{S} 2$ subunit to form the immune dominant, multifunctional, transmembrane moiety facilitating the viral attachment fusion and entry into the host cell) employs the same receptor, i.e., the human angiotensin-converting enzyme 2 (hACE2) and the 
same binding site (receptor-binding domain or RBD) as SARS-CoV applied on the host cell [3-5]. Moreover, approximately $79 \%$ genetic similarities have been noticed between the SARS-CoV and SARS-CoV-2 with a significant difference in the presence of the furin cleavage site (possibly conferring the COVID-19 severity upon viral pathogenesis) with the masked and glycan shielded $\mathrm{S}$ protein (which can also dodge the host protective immunity) of SARS-CoV-2, primed by the host transmembrane serine 2 (TMPRSS2) and the host endosomal cysteine proteases cathepsins B and L (CatB/L) $[3$, $4,6]$. However, the genetic resemblance mostly between the current SARS-CoV-2 and the previous version of this coronavirus, SARS-CoV (and with a little extent, around $50 \%$ on MERS-CoV which used dipeptidyl peptidase or DPP4 as the host receptor), may be useful in the development of effective vaccine against SARS-CoV-2 using any of the known platforms of vaccine preparation, i.e., (1) inactivated or live attenuated viruses (representative trials: NCT04412538 and NCT04324606, both in phase I), (2) the virus coated or protein subunit vaccines (representative trial NCT04405908 in phase II), (3) virus-like particles (VLP), (4) the replicating and nonreplicating viral vectors (adenovirus vaccines: trial NCT04341389 in phase II), (5) nanoparticles, (6) the DNA vaccines (representative trial NCT04368988 in phase I) or (7) RNA vaccines (representative trials: NCT04405076 and NCT04368728 in phase III and phase I, respectively), (8) application of oral delivery of plasmid constructs of the $\mathrm{S}$ protein, (9) adjuvant recombinant protein, and (10) the engineered bacterial vaccines with the principal aim to trigger the production of $S$ protein neutralizing antibodies [3, 6, 7].

Therefore, according to the ongoing COVID-19 vaccine development clinical trials, very few, especially the mRNA vaccines, are under phase III while other studies may need 3 years more [6]. The current review attempted to impart an update on the potential COVID-19 vaccine candidates with the success of the mRNA vaccines as the biologically licensed vaccines in the light of completed and ongoing clinical trials and to focus on the possible potentials of other vaccines as well. The associated risks upon vaccine administration have also been discussed.

\section{Potential Candidate Vaccine Candidates}

Prior to the discussion about the vaccines, it is to be recalled that there are three possible approaches for the treatment and prevention against COVID-19: (1) the immunomodulatory agents and the repurposed antiviral drugs (i.e., drugs that are already approved to treat other diseases such as the Ebola virus disease, AIDS, and malaria and to observe whether they are effective against infecting SARS-CoV-2 into the patients, like remdesivir, lopinavir, dexamethasone, and ritonavir); (2) introducing the COVID-19 patients with the antibodies (convalescent plasma therapy or the passive antibody therapy) either from the convalescent donors (patients who have recovered from the disease) or manufactured through the recombinant DNA technology; and (3) finally, by developing vaccines against the viruses using various platforms including the protein subunit vaccines, RNA vaccines, nonreplicating viral vaccines, attenuated viral vaccines, the virus-like particles, replicating viral vaccines, DNA vaccines, and the live attenuated vaccines (LAVs) which are currently under extensive research $[3,4,8$, 9]. Among the protein subunit vaccine candidates, the NVXCoV2373 (a nanoparticle-based immunogenic vaccine), the molecular clamp stabilized $\mathrm{S}$ protein vaccine conjugated with AS03 adjuvant system (a squalene-based immunogenic adjuvant instigating unique immunological profile within the vaccine on the basis of the immunological duration and strength), the microneedle array (MNA)-based recombinant immunogenic vaccine, and the multi-antigenic (triple antigen) virus-like particle (VLP) vaccines are significant for the enrolment to the clinical trials [3]. The viral vectored vaccines include the recombinant, replication-defective adenovirus type-5 vector (Ad5)-nCoV which can express the recombinant $\mathrm{S}$ protein, the LV-SMENP-DC vaccine (which can activate the host cytotoxic T cells against SARS-CoV-2), and the ChAdOx1 vaccine (discussed in the succeeding text). The live attenuated vaccine (LAV) known as the DelNS1-SARS-CoV-2-RBD is also significant which can express the receptor-binding domain (RBD) domain of SARS-CoV-2 spike protein [3].

Indeed, the different categories of COVID-19 vaccines have been elaborately discussed in several reports [3, 6, 7, 10-12]. In this review, the promising candidates which may be commercially applied for the quick mitigation of the disease are deliberated. For example, the viral vectored vaccines may instigate the elevation of cytotoxic T cells (CTL) which in turn eliminate the infecting SARS-CoV-2 particles, and hence, such vaccines are significant for prophylactic use [3]. The recombinant nonreplicating adenovirus type-5 (Ad5)-vectored vaccine has been reported to undergo the first randomized controlled trial (currently in phase II) in Wuhan, China [6, 7]. The outcome was that the administration of $5 \times 10^{10}$ viral particles appeared to induce the required host immunity together with safety [7]. The humoral and cellular immunity has been noticed to be triggered in the participants by the chimpanzee adenovirus vector vaccine, ChAdOx1 nCoV-19 (AZD1222), expressing the SARS-CoV-2 spike protein (Fig. 1), which underwent a phase I/II, single-blind, randomized controlled trial NCT04324606 in the UK, and was found to be safe and immunogenic with mild reactogenicity $[6,10]$. The instigation of (1) cellular immunity by enumerating the antigen-specific $\mathrm{T}$ cells and (2) the humoral responses noticed through the elicitation of IgG were assessed by the standard serological techniques as described by several groups $[6,7,10]$. Another promising vaccine developed in Russia (phase III clinical trial), the Gam-COVIDVac-Lyo vaccine, has been authorized for emergency use [6]. However, the DNA- and RNA-based approaches possess the greatest potential for escalating the development of effective 


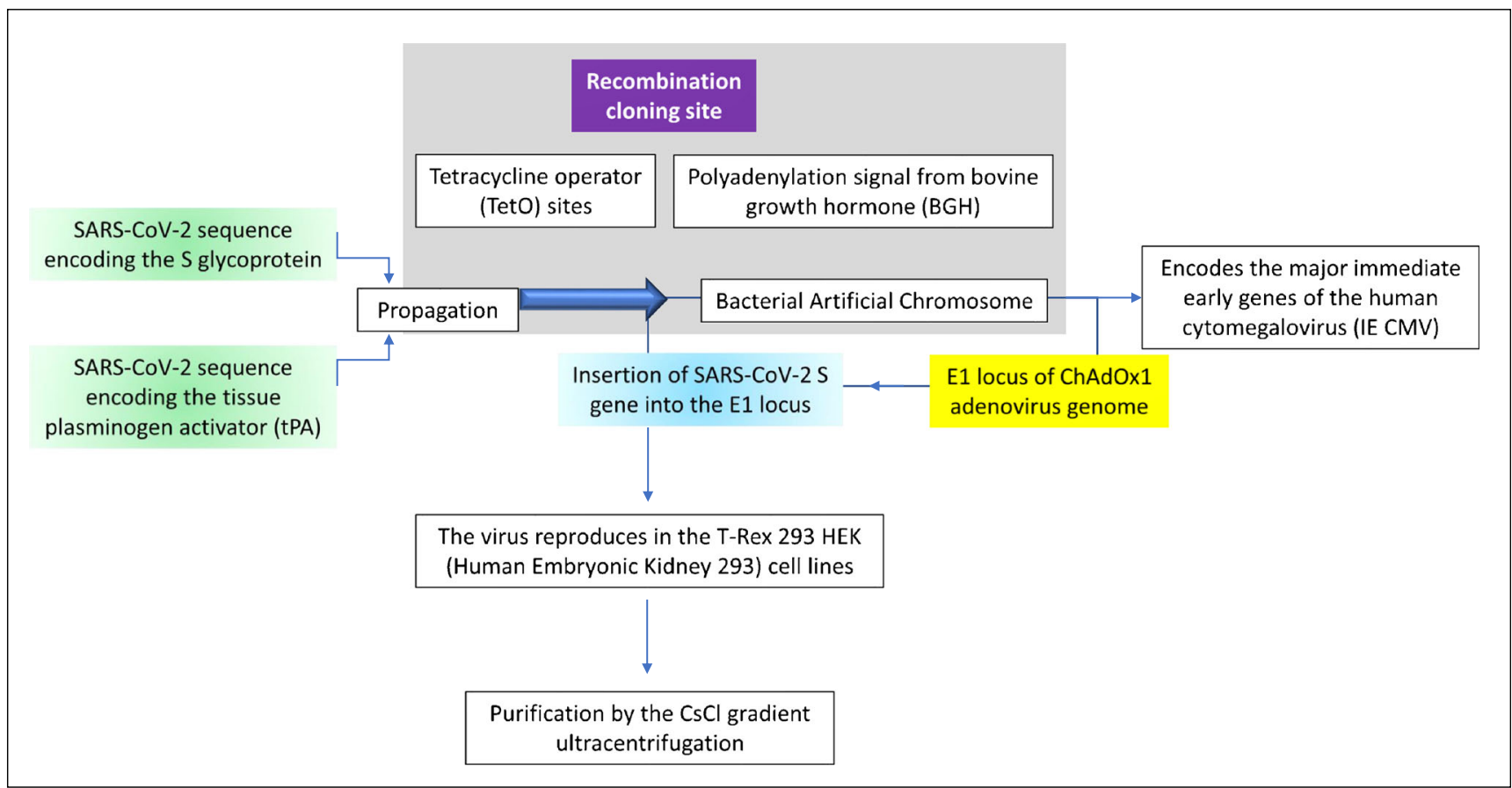

Fig. 1 Scheme of developing the ChAdOx1 recombinant adenovirus vaccine (adapted from the description reported by Kaur and Gupta) [3]

vaccines in the current dreadful situation caused by the SARSCoV-2 [11, 12]. A glimpse of these most prospective vaccines is given in the succeeding text.

\section{mRNA Vaccines}

This is to be noted in the current pandemic situation that the US Food and Drug Administration (USFDA) approved the use of the Pfizer-BioNTech (BNT162b2 vaccine) and Moderna vaccine (mRNA-1273 vaccine), both of which are the mRNA vaccines (whereby the SARS-CoV-2 spike glycoprotein (S) antigen is encoded by the mRNA and then devised in the lipid nanoparticles or the LNPs) with more than $90 \%$ effectiveness in candidates 16 years of age or older with 2 days within 21 days [13-15]. Descriptively, the mRNA-1273 vaccine (whereby the mRNA encoding the $\mathrm{S}-2 \mathrm{P}$ antigen, containing the SARS-CoV-2 glycoprotein attached to a transmembrane anchor, and an intact S1-S2 cleavage site, and is enclosed by a 4 subunit lipid nanoparticle) which has undergone the first-inhuman dose-dependent ( 25 or 30, 100, and $250 \mu \mathrm{g}$ ), open-label phase II clinical trial NCT04283461 (with the fast-track approval from the USFDA as stated above) successfully detected the elevated neutralizing antibody titers and the required $\mathrm{T}$ cell responses $[3,6,11,13]$. A unique characteristic of this vaccine is that this one is free from any preservative [12]. This is also notable that in the current phase III trial, around 44,000 participants are being subjected for the evaluation of the safety and efficacy of the mRNA-1273 (Moderna TX, Inc.) vaccine, and the outcome is $95 \%$ satisfactory so far [13].

\section{How Does mRNA-1273 Vaccine Work?}

The chemistry of the mRNA-1273 (Moderna TX, Inc.) vaccine is based on the contentment of (1) the nucleosidemodified messenger RNA (modRNA), i.e., the mRNA (as stated earlier) as the active ingredient that encodes the viral spike glycoprotein (S) of SARS-CoV-2 (i.e., RNA serves as the template to generate the specific protein that triggers the host immune response against the virus); (2) lipid nanoparticles (LNPs) with complexed polyethylene glycol (PEG), phosphocholine, and cholesterol (this is to be noted here that BioNTech-Pfizer and Moderna encapsulate their mRNA vaccines within LNPs which aids in the delivery of the RNA and protects the RNA from degradation); (3) salts including potassium chloride and sodium chloride, and monobasic potassium phosphate and the dibasic sodium phosphate dihydrate which serve as the buffer; and (4) sucrose, which acts as the cryoprotectant assuring that the lipid does not get too much sticky at the extremely cold temperature during storage [13]. The development procedure of the mRNA-1273 vaccine has been very well described by Corbett et al. [14]. Briefly, a DNA fragment consisting of the immunogen open reading frame (ORF) flanked by $5^{\prime}$ untranslated region (5' UTR) and the $3^{\prime}$ UTR is used as a template on which the T7 RNA polymerase mediates transcription to generate the mRNA encoding SARSCoV-2 S (2P) protein (Fig. 2), followed by the enzymatic addition of the cap structure and the subsequent purification of the mRNA. For encapsulation of the preclinical mRNA into the LNPs, the modified ethanol-drop nanoprecipitation process is applied whereby PEGs are mixed with the mRNA 


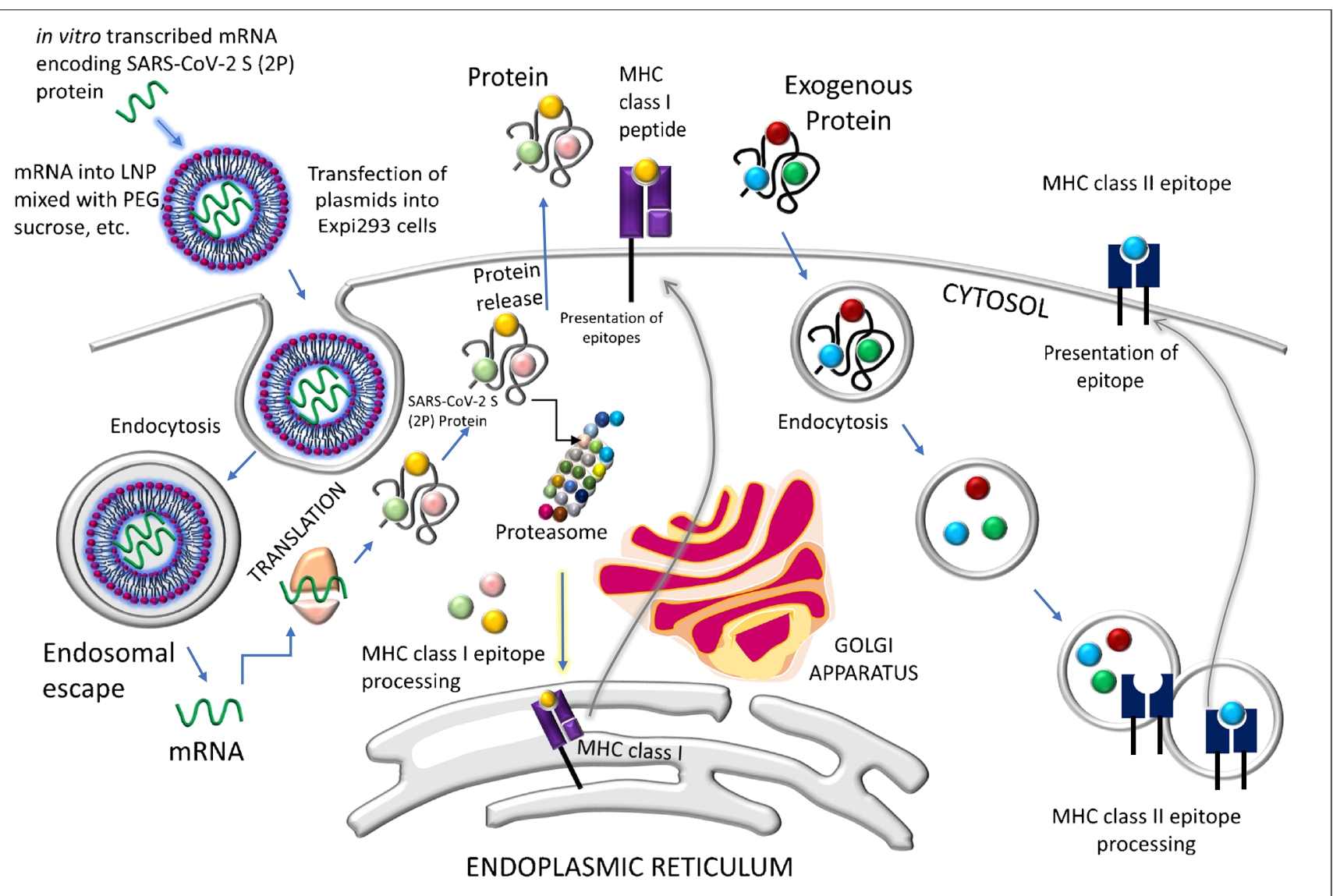

Fig. 2 A model showing the scheme of the mechanisms of action of the mRNA-1273 vaccine (adapted from the description reported by Corbett et al.) [11]. Details are described in the text. Briefly, following transfection and endocytosis, the mRNA is translated within the host to

(maintaining $\mathrm{pH}$ at 5.0 in the acetate buffer), neutralized, and finally, sucrose is added as a cryoprotectant as stated above, and the final solution is filter sterilized $[15,16]$. Prior to use, vials filled with the formulated LNP are stored in $-70^{\circ} \mathrm{C}$, and before the in vivo application, the product is assessed for a range of variables as described recently [14].

The MERS-CoV S-2P and the SARS-CoV S-2P proteins are expressed by the transfection of plasmids into Expi293 cells following the protein purification $[14,15]$.

As described by Corbett et al., the mammalian expression vector derived from pLEXm is used whereby a mammalian codon-optimized plasmid encoding foldon inserted minifibritin with a $\mathrm{C}$-terminal thrombin cleavage site is inserted following the expression of the construct by transient transfection of Expi293 [14]. In addition to the HEK293T/17 cells (transiently transfected with the mRNA that encodes the SARS-CoV-2 S or 2P protein) and the ACE2-expressing $293 \mathrm{~T}$ cell cultivation, the Vero E6 cells, in the plaque reduction neutralization test (PRNT), are used in plaque assays to detect the lung and nasal turbinate viral titers [14]. make SARS-CoV-2 S protein, which is released and also undergoes the MHC class I processing for the antigenic presentation on the host cell surface. The exogenous protein undergoes endocytosis followed by processing by MHC class II

\section{Effectiveness of the Pfizer-BioNTech and Moderna mRNA Vaccines}

Indeed, still, there are many unknowns regarding the SARS$\mathrm{CoV}-2$ which are resisting to encounter the ongoing disastrous pandemic even the preventive measures suggested by the World Health Organization (WHO) are being followed more or less all over the world. Apparently, the situation appears as an irresistible state, and the suppression of the COVID-19 transmission seems to be possible only when the herd immunity (i.e., the resistance against the viral spread within a population and a sufficient population may trigger the protective immunity against SARS-CoV-2 thereby reducing the probability of transmission between both the infected and susceptible individuals) will develop [17, 18]. Actually, until and unless any suitable vaccine is prepared, there will not be any improvement of the current pandemic situation. So far, nearly thirty 30 pharmaceutical companies and research institutes/academic organizations around the world have been engaged in developing the accurate vaccine against COVID-19; among them, Pfizer-BioNTech and Moderna appeared as the most successful in constructing vaccines using nanotechnology with the mRNA platform, and 
those under-trial vaccines (as stated above) have already shown required immunogenicity and safety according to the clinical trials [17]. The clinical trials conducted by Moderna reached the successful production of mRNA-1273 vaccine within a very short time (around 2 months) which can be compared with the previous SARS-CoV-1 and MERS-CoV clinical trials requiring around 2 years in both cases [17].

BioNTech/Pfizer, another leading pharmaceutical company, developed the SARS-CoV-2 vaccine (namely, BNT162b1) as stated previously which is actually a lipidnanoparticle-formulated (Fig. 2), nucleoside-modified mRNA vaccine encoding the receptor-binding domain (RBD) of the $\mathrm{S}$ protein of the virus. The vaccine is also currently under the dose-dependent $(10,30$, or $100 \mu \mathrm{g})$ clinical trial (ClinicalTrials.gov identifier NCT04368728), and the outcome seems to be satisfactory as the RBDbinding $\operatorname{IgG}$ concentrations and the SARS-CoV-2 neutralizing antibody titers in sera were noticed to be elevated [13, 18]. However, despite such fast progress by PfizerBioNTech and Moderna, some adverse effects of the vaccination in the trial stage have been noticed as the onset of fatigue, headache, muscle and joint pains, chills, fever, and, in some cases of the participants, the lymphadenopathy [13]. However, the high reported efficacy of the mRNA1273 vaccine is supported by the trial assay sensitivity which in turn in association to the industrial manufacturing (safe mRNA-LNP commercial mRNA vaccine delivery platform as shown in Fig. 2) of the established vaccine with required logistics and thereby facilitating a quick response to the current pandemic [14].

\section{DNA Vaccine}

The DNA vaccine, which induces both humoral and cellmediated immunity, is made on the principle that the genetic material of the SARS-CoV-2 is translocated (the antigenpresenting cells or APCs receive the genetic material) to the host's cell nucleus so that the mammalian promoter present in the plasmid vector is activated, triggering the transcription and translation of the transfected gene(s) within the host [12]. The resulting vaccine antigenic protein can then be presented to the APCs (mainly the DCs) through the MHC signaling: (1) the $\mathrm{CD} 8^{+} \mathrm{T}$ cell immunity is induced by the myocytes (MHC I signaling) which in turn stimulates the excretion of interferon (IFN)- $\gamma$ and the tumor necrosis factor (TNF)- $\alpha$, and thus, the viral replication inside the host is hindered (Fig. 3) and (2) the $\mathrm{CD}^{+}$helper $\mathrm{T}$ cells are activated by macrophages (MHC II signaling), and the DCs (MHC II signaling) activate the CD8+ $\mathrm{T}$ cells by triggering the production of interleukin (IL)-10, IL12 , and the tumor necrosis factor (TNF)- $\alpha$, and the $\mathrm{CD} 4^{+}$ helper $\mathrm{T}$ cells are activated by these DCs (Fig. 3 ) when they produce IL-4 $[12,16,19]$. This is to be mentioned that

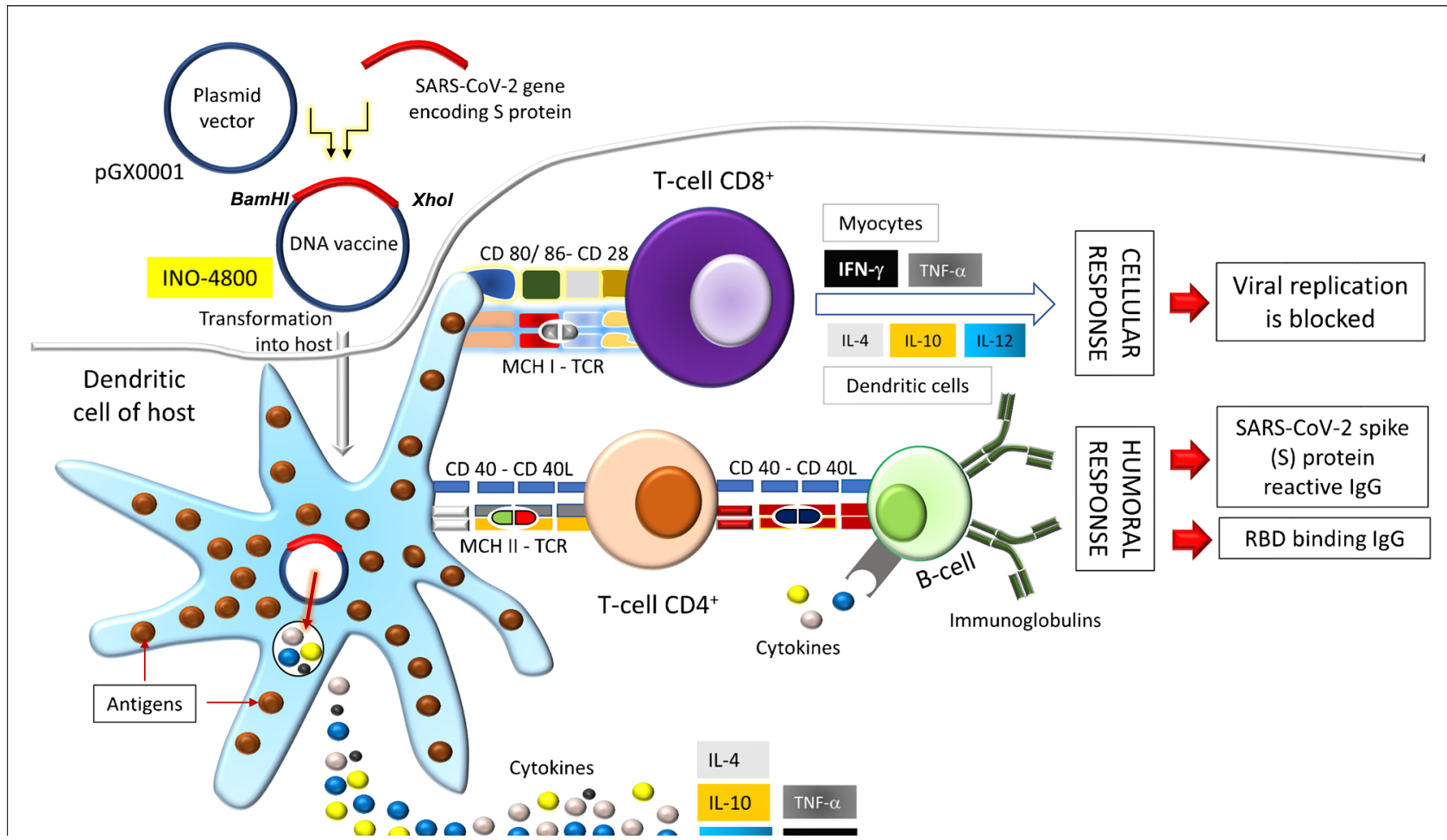

Fig. 3 A model showing the induction of cellular and humoral immunity with the action of the DNA vaccine INO-4800 (adapted from the description reported by Silveira et al.) [12]. Details are given in the text 
myocytes have been evidently the predominant transfected cell type triggering the potential cell-mediated immunity [20]. The antigen transfer from myocytes to the professional APCs is thus significant for the induction of the cytotoxic T cells through the administration of DNA vaccines [20].

The vaccine protein serves as the target for the recognition by the $\mathrm{B}$ cell receptor, and the activated $\mathrm{B}$ cells may produce principally IgG [12]. INO-4800, which is now under the phase I clinical trial (NCT04336410), is a prophylactic DNA vaccine (Fig. 3) which is prepared on the basis of the codonoptimized S protein sequence (synthesized and digested using BamHI and XhoI) of the virus to which an IgE leader sequence is affixed and the digested DNA is incorporated into the expression plasmid pGX0001 which in turn elicits the production of S protein reactive (both $\mathrm{S} 1$ and $\mathrm{S} 2$ ) and the RBD-binding IgG and the $T$ cell responses $[3,21]$.

\section{Comparative Efficacy Between the DNA and RNA Vaccines and the Commencement of RNAi Therapy and CRISPR-Cas13-based Antiviral Strategy}

Considering the instability of mRNA and its immunostimulatory nature (as an agonist of the toll-like receptor, TLR3), DNA vaccines may apparently be preferable; however, using pseudouridine instead of uridine may generate the stable form of mRNA which is really effective in the simultaneous translation of the SARS-CoV-2 S protein together with decreased immunogenicity upon administration within the LNPs into the host [13, 22]. In addition, compared to the plasmid DNA vaccines, whose entry into the host nucleus is obvious or necessary to produce mRNAs, the administration of mRNA vaccines easily permits the mRNA directly to be placed in the cytoplasm thereby increasing the translation frequency [22]. However, based upon the chemistry and the mode of action of these two types of vaccines, the optimal and intentional delivery with precise formulations of either the DNA vaccine or the mRNA vaccine may bring about the similar benefits to the patients. Interestingly, in order to mitigate the COVID-19 pandemic, besides the ongoing efforts including the vaccination strategies, convalescent plasma therapy, intravenous immunoglobulin (IVIG) infusion therapy; application of ACE-2 inhibitors and the angiotensin 1 receptor (AT1R), hindering the hACE2-SARS-CoV-2-RBD interaction by the usage of monoclonal antibodies; SARS-CoV RNA-dependent RNA polymerase (RdRp) targeting drugs, repositioned antivirals and the immunomodulatory agents; the RNA interference (RNAi) therapy has also got attention especially if the inadvertent mRNA or the DNA vaccination outcome arises [23]. As elaborately discussed by Sarwar et al., due to the RNAi-provoked silencing, (1) the SARS-
CoV-2 nonstructural protein 15 (nsp15) imparting the antiapoptosis trait, (2) the ORF-3a and ORF-4a (responsible for the viral release within the infected individual), and (3) the ORF-9b (the IFN agonist) may become the possible targets of the RNAi therapy, and hence, the viral life cycle within the host can be blocked [23]. Besides, three noncoding microRNAs (miR-1246), which can act as the RNAi-targets, have been noticed to curb the expression of ACE-2 [23]. Another antiviral strategy can be the employment of the CRISPR-Cas13-based strategy across the human respiratory tract whereby the prophylactic antiviral CRISPR (PAC-MAN) can hinder the viral replication and release by degrading the viral RNA [23, 24]. CRISPR RNAs (crRNAs) can target the SARS-CoV-2 conserved genomic regions, and a bioinformatic analysis by Abbott et al. showed that a group of only six crRNAs were more than $90 \%$ effective as anti-SARS-CoV-2 agents even though the synthesized fragments were used [24]. However, it is speculated that the RNAi therapy or the CRISPR-based therapy would be useful if new mutations arise in the viral spike protein-encoding genomes (already 15 amino acid variations have been observed in the RBD of the mutant SARS-CoV-2) which may render the mRNA vaccines or the DNA vaccines inactive against the new strains of SARS-CoV-2 [25, 26]. Extensive clinical trials using these two new approaches would be required to prove such an assumption though.

\section{Conclusion}

Several research teams have been engrossed although 2020 in dissecting innumerable antiviral drugs for their usefulness and doing the best for developing diverse forms of vaccines through a number of clinical trials with notable success imparting long-term immunity against SARS-Co-V. Besides the nearly successful mRNA-1273 vaccine which is expected to be launched commercially for the mitigation of the COVID19 pandemic by 2021 , still there are requirements for the development of other successful and licensed vaccines with satisfactory clinical trials regarding vaccine efficacy, safety, and justified logistics. A sound and flawless platform of clinical trials imparting the nonbiased data on the vaccine efficacy together with a comparative valuation of the accredited vaccines would open up the quick and the finest way to develop new vaccines against SARS-CoV-2. However, one point is to ponder that viruses can mutate, and if a large-scale mutation occurs within the spike protein of the viral inoculum within the vaccine, it would not trigger the cellular or humoral immune response in the host. Hence, regular analysis of the genome sequences is another necessary part not only in course of vaccine development phases but also to maintain vaccine sustainability. Moreover, due to the evidence of the rising 
variants of SARS-CoV-2, replacing the current vaccine's S protein with the renovated molecule with the required changes in the specific amino acid (with a concomitant observation of the host protective immunity against the new version of the vaccine) or by adding the new molecule to the existing formulation (i.e., forming the multivalent vaccine) would be a choice to effectively combat the upcoming SARS-CoV-2 infection. Following the current clinical trials, if the current vaccine does not seem to be effective against the new variants, the formulation and implementation of the seasonal vaccine may be required.

Author Contribution RN: conceptualization; literature survey; formal analysis; investigation; resources; writing-original draft preparation; editing; visualization; and supervision.

Data Availability Not applicable.

Code Availability Not applicable.

\section{Compliance with Ethical Standards}

Competing Interests The author declares no competing interests.

Animal Research Not applicable.

Consent to Participate Not applicable.

Consent to Publish The author read and approved the final manuscript for publication.

\section{References}

Papers of particular interest, published recently, have been highlighted as:

- Of importance

•- Of major importance

1. Noor R, Maniha SM. A brief outline of respiratory viral disease outbreaks: 1889 - till date on the public health perspectives. VirusDis. 2020;31:441-9. https://doi.org/10.1007/s13337-02000628-5.

2. World Health Organization (WHO) coronavirus disease (COVID19) dashboard. Data last updated: 2020/12/26, 5:03pm CET. https://covid19. who.int/?gclid=CjwKCAiA25v_BRBNEiwAZb4ZYq8KNQr8xNMczDO3hKJosdVab_SLoPTlcWwUXyGzc4uX3TV31-FxoC6uUQAvD BwE Accessed on 2020/12/27.

3. Kaur SP, Gupta V. COVID-19 vaccine: a comprehensive status report. Virus Res. 2020;288:198114. https://doi.org/10.1016/j. virusres.2020.198114

4. Noor R. Antiviral drugs against severe acute respiratory syndrome coronavirus 2 infection triggering the coronavirus disease-19 pandemic. Tzu Chi Med J. 2020;33:7-12. https://doi.org/10.4103/tcmj. tcmj_100_20.
5. Asaduzzaman SAI, Zakaria A, Kheya IS, Fahad N, Sikandar YB, Noor R. A comparative study between the severe acute respiratory syndrome-coronavirus-2, severe acute respiratory syndrome coronavirus, and the Middle East respiratory syndrome coronavirus. Biomed Biotechnol Res J. 2020;4:S65-74. https://doi.org/10. 4103/bbrj.bbrj_99_20.

6. TofailAhmed Raja A, Alshamsan A, Al-Jedai A. Status of the current COVID-19 vaccine candidates: implications in the Saudi population. Saudi Pharm J. 2020;28:1743-8. https://doi.org/10.1016/j. jsps.2020.10.019.

7. Zhu FC, Guan XH, Li YH, Huang JY, Jiang T, Hou LH, et al. Immunogenicity and safety of a recombinant adenovirus type-5vectored COVID-19 vaccine in healthy adults aged 18 years or older: a randomised, double-blind, placebo-controlled, phase 2 trial. Lancet. 2020;396(10249):479-88. https://doi.org/10.1016/S01406736(20)31605-6.

8. Sikandar YB, Shabnam I, Noor R. Remdesivir and dexamethasone: the two eligible candidate drugs against severe acute respiratory syndrome coronavirus 2 (SARS-CoV-2) infection. Biomed Res J. 2020. https://doi.org/10.4103/BMRJ.BMRJ_10_20.

9. Altuntas F, Ata N, Yigenoglu TN, Bascı S, Dal MS, Korkmaz S, et al. Convalescent plasma therapy in patients with COVID-19. Transfus Apher Sci. 2020;102955:102955. https://doi.org/10. 1016/j.transci.2020.102955.

10. Folegatti PM, Ewer KJ, Aley PK, Angus B, Becker S, BelijRammerstorfer S, et al. Oxford COVID Vaccine Trial Group. Safety and immunogenicity of the ChAdOx $1 \mathrm{nCoV}-19$ vaccine against SARS-CoV-2: a preliminary report of a phase $1 / 2$, singleblind, randomised controlled trial. Lancet. 2020;396(10249):46778. https://doi.org/10.1016/S0140-6736(20)31604-4.

11. Jackson LA, Anderson EJ, Rouphael NG, Roberts PC, Makhene M, Coler RN, et al. An mRNA vaccine against SARS-CoV-2 - preliminary report. N Engl J Med. 2020;383(20):1920-31. https://doi.org/ 10.1056/NEJMoa2022483.

12. Silveira MM, Moreira GMSG, Mendonça M. DNA vaccines against COVID-19: perspectives and challenges. Life Sci. 2020;267:118919. https://doi.org/10.1016/j.lfs.2020.118919.

13. Vaccines and Related Biological Products Advisory Committee Meeting December 10, 2020. FDA briefing document: PfizerBioNTech COVID-19 vaccine. https://www.fda.gov/media/ 144245/download Accessed on December 20, 2020.

14. Joffe S. Evaluating SARS-CoV-2 vaccines after emergency use authorization or licensing of initial candidate vaccines. JAMA. . https://doi.org/10.1001/jama.2020.25127.

15. Corbett KS, Edwards D, Leist SR, Abiona OM, Boyoglu-Barnum $\mathrm{S}$, Gillespie RA, et al. SARS-CoV-2 mRNA vaccine development enabled by prototype pathogen preparedness. Nature. 2020;586: 567-71. https://doi.org/10.1038/s41586-020-2622-0.

16. Wrapp D, Wang N, Corbett KS, Goldsmith JA, Hsieh CL, Abiona O, et al. Cryo-EM structure of the 2019-nCoV spike in the prefusion conformation. Science. 2020;367(6483):1260-3. https:// doi.org/10.1126/science.abb2507.

17. Chung YH, Beiss V, Fiering SN, Steinmetz NF. COVID-19 vaccine frontrunners and their nanotechnology design. ACS Nano. 2020;14(10):12522-37. https://doi.org/10.1021/acsnano.0c07197.

18. Mulligan MJ, Lyke KE, Kitchin N, Absalon J, Gurtman A, Lockhart S, et al. Phase I/II study of COVID-19 RNA vaccine BNT162b1 in adults. Nature. 2020;586(7830):589-93. https://doi. org/10.1038/s41586-020-2639-4.

19. Duerr GD, Heine A, Hamiko M, Zimmer S, Luetkens JA, Nattermann J, et al. Parameters predicting COVID-19-induced myocardial injury and mortality. Life Sci. 2020;260:118400. https://doi.org/10.1016/j.lfs.2020.118400. 
20. Donnelly JJ, Liu MA, Ulmer JB. Antigen presentation and DNA vaccines. Am J Respir Crit Care Med. 2000;162(4 Pt 2):S190-3. https://doi.org/10.1164/ajrccm.162.supplement_3.15tac10.

21. Smith TRF, Patel A, Ramos S, Elwood D, Zhu X, Yan J, et al. Immunogenicity of a DNA vaccine candidate for COVID-19. Nat Commun. 2020;11(1):2601. https://doi.org/10.1038/s41467-02016505-0.

22. Liu MA. A comparison of plasmid DNA and mRNA as vaccine technologies. Vaccines (Basel). 2019;7(2):37. https://doi.org/10. 3390/vaccines7020037.

23. Sarwar Z, Ahmad T, Kakar S. Potential approaches to combat COVID-19: a mini-review. Mol Biol Rep. 2020;47(12):9939-49. https://doi.org/10.1007/s11033-020-05988-1.

24. Abbott TR, Dhamdhere G, Liu Y, Lin X, Goudy L, Zeng L, et al. Development of CRISPR as an antiviral strategy to combat SARS-
CoV-2 and influenza. Cell. 2020;181(4):865-876.e12. https://doi. org/10.1016/j.cell.2020.04.020.

25. Durmaz B, Abdulmajed O, Durmaz R. Mutations observed in the SARS-CoV-2 spike glycoprotein and their effects in the interaction of virus with ACE-2 receptor. Medeni Med J. 2020;35(3):253-60. https://doi.org/10.5222/MMJ.2020.98048.

26. Xia Y, Zhong L, Tan J, Zhang Z, Lyu J, Chen Y, et al. How to understand "herd immunity" in COVID-19 pandemic. Front Cell Dev Biol. 2020;8:547314. https://doi.org/10.3389/fcell.2020. 547314.

Publisher's Note Springer Nature remains neutral with regard to jurisdictional claims in published maps and institutional affiliations. 